\title{
Prolidase Deficiency: A child with persistent lower extremity ulcerations
}

\author{
by Morris Tyrone Haywood, DPM ${ }^{1 ⿴}$ \\ The Foot and Ankle Online Journal 4 (4): 4
}

This case report describes a pediatric patient with prolidase deficiency, a rare, autosomal recessive metabolic disorder with severe dermatological manifestations, particularly ulcers of the lower extremities. While this condition has seldom been reported, it may be more common than previously acknowledged. An eight year-old Amish girl with prolidase deficiency presents with ulcerations on the feet and lower legs. Clinical presentation and the pathogenesis of prolidase deficiency are discussed. During the past five years various treatment options have been attempted. These have included ointments containing growth hormones, amino acids, antibiotics and numerous over the counter products. Skin grafts were also attempted for this patient. To date, no treatment has proved significantly successful. Currently there is no cure for this condition.

Key words: Energy consumption, First metatarsophalangeal joint, First metatarsal, Insolia ${ }^{\circledR}$ Flex, Comfort.

$\mathrm{P}$ rolidase deficiency (PD) is a rare, inherited, autosomal recessive, inborn error of amino acid metabolism that affects collagen maturation. Eighty-five percent of the patients present with dermatological symptoms, including lower extremity ulcers, with pedal ulcers as the common clinical finding at diagnosis in $50 \%$ of the patients. ${ }^{1} \quad$ These chronic leg ulcers are usually irregularly shaped with prominent granulation tissue. They are often resistant to various topical, systemic, and surgical treatments making them slow to heal. ${ }^{2}$

\footnotetext{
Address Correspondence to: Morris T. Haywood, DPM. Ohio College of Podiatric Medicine, 16000 Rockside Woods Blvd N.,Independence, Ohio 44131-2330

1 Ohio College of Podiatric Medicine, 16000 Rockside Woods Blvd N.,Independence, Ohio 44131-2330
}

In 1968, Goodman, et al., were the first to describe prolidase deficiency. ${ }^{3}$ Powell, et al., in 1974 further defined the clinical characteristics by documenting the absence of prolidase enzyme in association with the characteristic clinical features of prolidase deficiency. ${ }^{4}$ Prolidase enzyme cleaves iminodipeptides with Cterminal proline or hydroxyproline. The gene encoding this enzyme is located at chromosome 19. Prolidase deficiency has an incidence of 1-2 per $1,000,000$ persons with an estimated 60 cases described in the literature. ${ }^{5}$ 


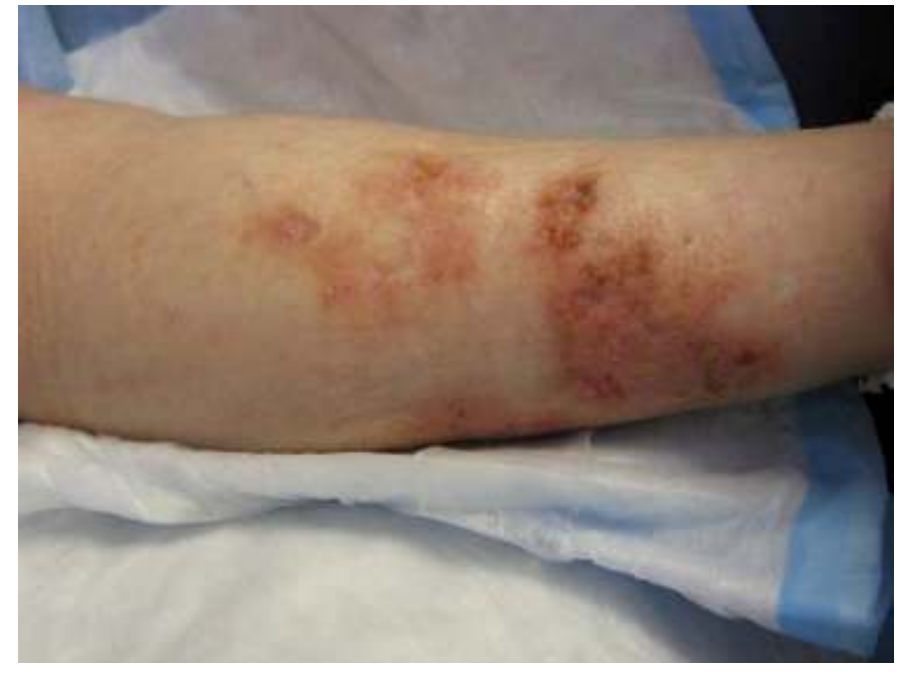

Figure 1 Irregularly shaped, intact skin with subcutaneous micro-vascular bleeding lesions.

The clinical features observed with prolidase deficiency vary from asymptomatic to a range of symptoms, including chronic leg ulcers, recurrent infections, characteristic facies, mental retardation, and splenomegaly. ${ }^{4}$ These symptoms normally occur in the first year of life but can remain dormant in an affected individual until the second decade of life. ${ }^{6,7}$

Prolidase deficiency affects the normal physiology of dermal collagen and skin. ${ }^{6}$ Collagen is degraded through several steps to iminodipeptides that are then split to free amino acids by the action of prolidase and other dipeptidases. These amino acids are resynthesized to form new collagen without readily exchanging with those in the general systemic pool. In a prolidase deficient state, iminodipeptides with Cterminal proline or hydroxyproline cannot be split. This causes an abnormal amount of glycine and proline dipeptides comprising over $30 \%$ of the amino acids in the normal dermal layer of collagen to be excreted in the urine and removed from the collagen cycle. Dermal layer collagen synthesis without adequate amounts of proline and glycine causes an irregular cross linking, an atypical basal lamina, and excessive high levels of type III collagen., ${ }^{3,8}$ Subsequently, the dermal collagen produced in patients with PD has a fragile and dysfunctional structure.
In acute ulcerations, vascular changes including occlusion of medium-sized vessels with amyloid deposits and perivascular infiltration of neutrophils have been observed.' Additionally, Pierand, et al., cited vascular wall thickening and infiltration of mononuclear cells and neutrophils in pre-ulcerative indurated lesions. ${ }^{10}$ Yasuda, et al., believed the difference in the severity of skin lesions and the mental retardation between two patients may have been related to the relative superoxide-generating activity of polymorphonuclear cells (PMN). ${ }^{11}$ These vascular and tissue changes result in chronic lower extremity ulcerations and other dermatological conditions, including scarring, scaly abnormally thick skin, photosensitivity telangiectasia, poliosis, and lymphedema. ${ }^{12}$ Researchers have yet to determine why these chronic ulcers occur mainly in the lower extremities. ${ }^{13}$

\section{Case Report}

An 8 year-old Amish girl (patient x) presented with a six year history of lower extremity ulcerations, primarily on her feet. Recently, the number of ulcerations has increased, causing her more pain and discomfort. The patient's past medical history is significant for chronic lower extremity ulcers associated with prolidase deficiency.

A physical examination of the lower extremity revealed multiple lesions in various stages of healing on her feet and legs. (Figs.1, 2A AND 2B and 3) The ulcerations on the right foot also had diffuse dyspigmentation, erythema and scaly skin. (Fig. 3) However, digital clubbing was more visible on her hands compared to her feet. She was in significant pain with ambulation and compensated by toe walking. Her parents confirmed that their child is still primarily a toe walker to avoid plantar foot pressure. Both of the patient's feet rest in a pronounced equinus type position non- weightbearing. The patient uses bactroban and topical zinc daily on the ulcers and takes Tylenol \#3 for the foot pain. Although flexible, the patient has dorsiflexion at the ankle that can reach only $90^{\circ}$ with effort and some discomfort. 


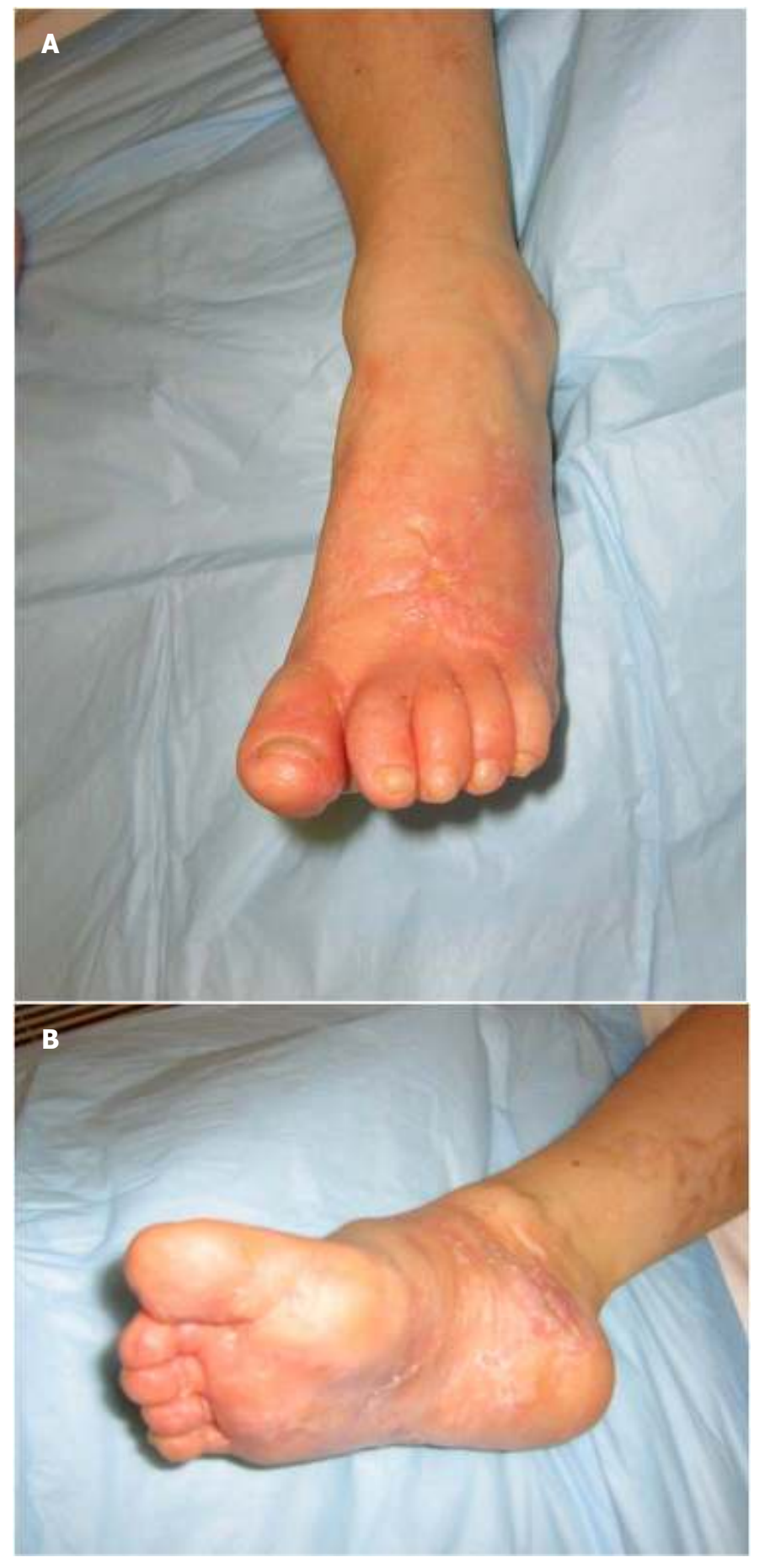

Figures 2A and 2B Dorsal left foot with erythema, edema at digits and scar tissue from healed ulcerations. (A) Plantar right foot with scaly erythematous skin. (B)

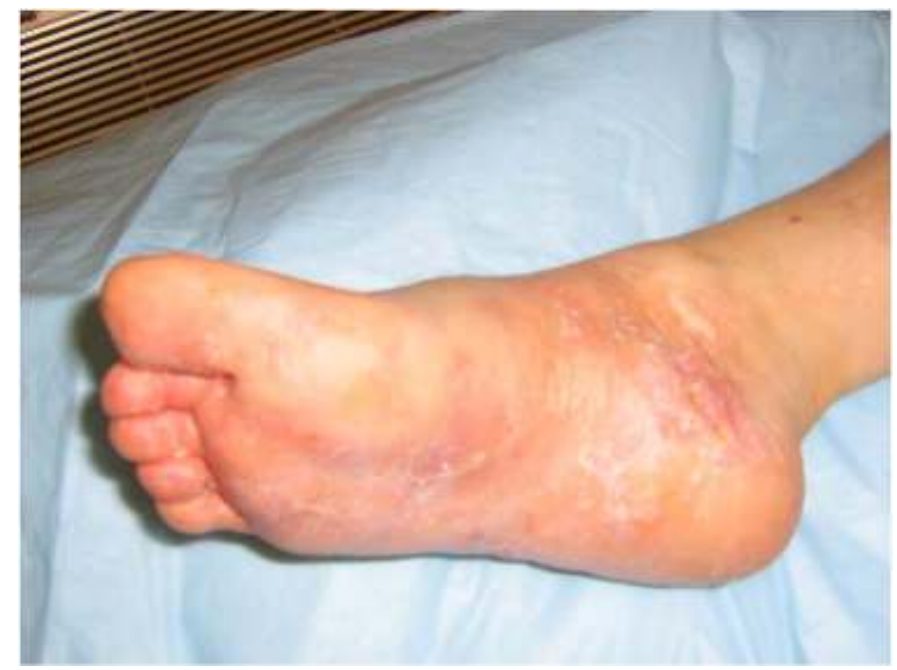

Figure 3 Close-up of plantar right foot with scaly erythematous skin.

The patient has tight Achilles tendons bilaterally. Additionally, the patient exhibits pronounced ligamentous laxity in many upper and lower body joints. For shoe gear, she wears a child's size 13 extra depth sneaker with velcro straps. The shoe also has an inner sole, comprised of a 1/16-inch pink plastazote cover on top of a 1/16-inch layer of white poron. At the heel plantarly, a small portion of NeoSponge covers the white poron.

In the first sixth months of her life, the patient was healthy and normal. However, at seven months, she developed petechia associated with anemia and thrombocytopenia. Then at age two, the patient was diagnosed with prolidase deficiency (PD) and underwent an allogenic umbilical cord blood transplant from her HLA identical sister to treat the disease. This initial treatment modality failed to resolve the patient's condition.

The patient's first lower extremity lesion was prior to the transplant. A superficial puncture wound resulted in a small, but difficult-to-heal lesion. Multiple lesions appeared on her feet and legs approximately 3 months after her transplant. These lesions became a chronic condition with the foot ulcers being most resistant to treatment and somewhat compromised by socks and shoe gear. 
These foot ulcers, which have been positive for fungus in the past, have resolved with using clotrimazole.

In regards to the patient's development, the mother had a full term delivery with no complications. The patient met all her milestones without much delay, except she began walking at around 18 months. Although she is within the appropriate percentile on the chart, she is short in stature which her primary care physician attributes to the prolonged use of steroids. However, the patient is not deficient in growth hormone as cited in the literature of other prolidase deficiency patients. She is very limber; in her mother's words the patient is very double-jointed.

Currently, the patient is seen approximately monthly in the Pediatric Hematology/ Oncology Clinic to receive intravenous immunoglobulin $G$ therapy to treat hypogammaglobulinemia. The patient also suffers with other immune-deficiency-related illnesses, including recurrent pulmonary and eye infections for which she often takes prophylactic medications. Additionally, she has reactive airway disease requiring the periodic use of rescue and preventive inhalers as well as a cystic fibrosis vest and oxygen. She also has a history of adrenal and thyroid deficiency involving periodic replacement. Finally, she has some developmental delay but has a 90 to 100 percent psychosocial performance score. Although none of the patient's siblings experienced similar medical problems, two paternal $1^{\text {st }}$ male cousins died from complications of PD at 13 months and 7 years of age.

\section{Diagnosing Prolidase Deficiency}

Diagnosis is based on decreased prolidase activity in erythrocytes, leukocytes and skin fibroblasts as well as urinary excretion of C-terminal proline and hydroxyproline iminodipeptides with glycylproline dominating. ${ }^{10} \quad$ Like most genetic disorders, PD can also be diagnosed in utero using amniocentesis or chorionic villus sampling.
Often laboratory abnormalities associated with prolidase deficiency include iron-deficiency anemia, thrombocytopenia, elevated liver enzymes, decreased growth hormone, and elevated serum immunoglobulins. ${ }^{10,14}$ However, the patient in this study has hypoimmuglobulin, requiring monthly replacement.

The low incidence and prevalence of PD and its range of phenotypes makes the diagnosis of PD a challenge. Signs and symptoms normally occur in the first year of life, but can remain latent until the second decade. ${ }^{15}$ This possible delay results in early symptoms of PD being linked to many medical conditions and diagnoses. Often patients with PD may struggle for many years to obtain the correct diagnosis. In most cases, PD patients receive multiple medical treatments for the chronic symptoms with providers having no real understanding of the disease process or the incurable prognosis. To date, therapeutic intervention of PD focuses on healing the chronic lower extremity ulcers with minimal attention given to the underlying etiology of PD. This lack of an accurate diagnosis lends patients to often feel disenfranchised in hoping for a cure for their disease.

\section{Difficulty in Treatment}

A characteristic feature of the lower extremity ulcers in this disorder is resistance to most forms of treatment, including rejection of skin grafts. The difficulty in treating PD lower extremity ulcers is probably related to the abnormal collagen degradation and synthesis cycle, which cause urinary excretion of proline and glycine as well as the vascular changes in ulcerated tissue. The collagen comprising the dermal layer is frail in PD, causing skin to break down from normal physiological stress. Furthermore, the wound healing process is altered, because of the tissue lacking normal tension, thus causing the patient to be prone to dehiscence. Additionally, occluded vessels and abnormal infiltration of neutrophils contribute to an altered immune response comprised wound healing. These tissue and vascular changes predispose patients with PD to numerous recalcitrant wounds. 


\section{Discussion}

During the past five years, the patient's parents in this study have tried numerous products for treatment of their daughter's lower extremity lesions. Some of these topical creams and ointments have been more beneficial than others. For instance, lidocaine ointment has been mixed with various other products to relieve pain. However, with each new product, some successes were gained for several months, then, as if her body had become accustomed to the medication, the lesions returned significantly worse or at least to the degree at the time treatment was initiated. For other ointments, a negative effect was sometimes immediately experienced by the patient or later developed.

Regranex, a growth hormone ointment FDA approved for diabetic ulcerations was used for one week before being discontinued because the patient experienced burning sensations. Several over the counter (OTC) ointments for treating burns and wounds were also tried, including B \& W Ointment, an organic Amish product comprised of lanolin and honey; Wound-Be-Gone, a hydrophilic gel; and Merpelex, an emulsifying wax and paraffin. Unfortunately, all of these OTC products made the lesions dry and caused painful fissures. Another local product Wood's Poultice made of bentonite clay and pine tar oils caused dry painful fissures on the feet but not the legs. Currently, the patient's parents are applying an inexpensive triple antibiotic with zinc oxide on the feet and Wood's Poultic to the legs. They have used this combination with daily dressing for over 12 months. Although this treatment regimen is not a cure, it keeps the lesions moist while slowly healing.

The literature has reported minimal results with other topical agents, vitamins, and antibiotics including dapsone, bactroban, ascorbic acid, and manganese. ${ }^{14}$ The use of systemic and topical growth hormones, oral vitamins, and other supplements has provided minimal relief for a brief time. Lastly, surgical intervention using skin grafts has also been performed with limited success.
Some therapies target the pathophysiology of the disease by replacing the PD enzyme in the blood and providing collagen products to the wound. Enzyme replacement has been tried through blood transfusions, containing manganese activated prolidase enzyme, yet this costly treatment resulted in only a brief elevation in prolidase activity level. ${ }^{16}$ There are some reports on the use of collagen dressings; however, most show no true benefits even though collagen products are thought to stimulate fibroblast activity and to improve the healing cascade. Finally, pulsed corticosteroid treatment has shown good results by inhibiting abnormal infiltration of neutrophils. ${ }^{11}$

While the patient's current treatment is helpful, a different regiment will be attempted when this particular approach is no longer satisfactory. A combination ointment of proline and glycine has been described several times in the literature as beneficial. ${ }^{17,18}$ The parent's of this patient made several attempts of applying a compounded topical combination ointment of 5\% proline and 5\% glycine. After ointment application, an Adaptic wound dressing and dry gauze was then applied. Surprisingly, this treatment also was unsuccessful for this patient. She complained the ointment either burned when applied or made her itch. The ointment was temporarily discontinued for further evaluation. In the near future this combination may be manufactured with smaller percentages and/or other products. Tendon contractures resulting in foot deformity has been cited in this patient population, usually as a consequence of chronic ulcerations and scare tissue. ${ }^{2}$ Additionally, the patient will possibly undergo an Achilles tendon lengthening procedure.

The main problem posed in the treatment of PD is that it is a rare disease, and therefore, attracts little interest from the pharmaceutical industry. ${ }^{16}$ However, ulcerations in many disease processes are still a challenge to treat in a reasonable time frame. As the technology in wound care advances for treating our most common lesions, hopefully the treatment of chronic ulcers in PD will also improve. 


\section{References}

1. Der Kaloustian VM, Freij BJ, Kurban AK. Prolidase deficiency: An inborn error of metabolism with major dermatological manifestations. Dermatology 164: 293-304, 1982. 2. Leoni A, Cetta G, Tenni R, Pasquali-Ronchetti I, Bertolini F, Guerra D, Dyne K, Castellani A. Prolidase deficiency in two siblings with chronic leg ulcerations: Clinical, biochemical, and morphologic aspects. Arch Dermatol 123: 493-499, 1987. 3. Goodman SI, Solomons CC, Muschenheim F, Mclntyre CA, Miles B, O'Brien D. A syndrome resembling lathyrism associated with iminodipeptiduria. Am J Med 45:152-159, 1968.

4. Powell GF, Rasco MA, Maniscalco RM. A prolidase deficiency in man with iminopeptiduria. Metabolism: Clinical Experimental 23: 505-513, 1974.

5. Kelly JJ, Freeman AF, Wang H, Cowen EW, Kong HH. An Amish boy with recurrent ulcerations of the lower extremities, telangiectases of the hands, and chronic lung disease. J Am Academy Dermatol 62: 1031-1034, 2010.

6. Lopes I, Marques L, Neves E, Silva A, Taveira M, Pena R, Vilarinho L, Martins E. Prolidase deficiency with hyperimmunoglobulin E: A case report. Pediatric Allergy Immunology 13: 140-142, 2002.

7. Shrinath M, Walter JH, Haeney M, Couriel, J M; Lewis, M A; Herrick, A L. Prolidase deficiency and systemic lupus erythematosus. Archives Disease in Childhood 76: 441-444, 1997.

8. Sekiya M, Ohnishi Y, Kimura K. An autopsy case of prolidase deficiency. Virchows Archiv Pathological Anatomy

Histopathology 406:1 25-131, 1985.

9. Ogata A, Tanaka S, Tomoda T, Murayama E, Endo F, Kikuchi I. Autosomal recessive prolidase deficiency: Three patients with recalcitrant leg ulcers. Arch Dermatol 117: 689-694, 1981.

10. Milligan A, Graham-Brown RAC, Burns DA, Anderson I. Prolidase deficiency: a case report and literature review. Brit J Dermatol 121: 405-409, 1989.

11. Yasuda K, Ogata K, Kariya K, Kodama H, Zhang J, Sugahara K, Sagara Y, Kodama H. Corticosteroid treatment of prolidase deficiency skin lesions by inhibiting iminodipeptideprimed neutrophil superoxide generation. Brit J Dermatol 141: 846-851, 1999.
12. Arata J, Umemura S, Yamamoto Y, Hagiyama M, Nohara N. Prolidase deficiency: Its dermatological manifestations and some additional biochemical studies. Arch Dermatol 115: 62-67, 1979. 13. Jackson SH, Dennis AW, Greenberg M. Iminodipeptiduria: a genetic defect in recycling collagen; a method for determining prolidase in erythrocytes. Canadian Med Assoc J 113: 762-763, 1975.

14. Bissonnette R, Friedmann D, Giroux JM, Dolenga M; Hechtman P; Der Kaloustian V M; Dubuc R. Prolidase deficiency: a multisystemic hereditary disorder. J Am Academy Dermatol 29(5 Pt 2): 818-821, 1993.

15. Klar A, Navon-Elkan P, Rubinow A, Branski D, Hurvitz H, Christensen E, Khayat M, Falik-Zaccai TC. Prolidase deficiency: it looks like systemic lupus erythematosus but it is not. European J Pediatrics 169(6):727-732, 2010.

16. Viglio S, Annovazzi L, Conti B, Genta I, Perugini P, Zanone C, Casado B, Cetta G, Iadarola P. The role of emerging techniques in the investigation of prolidase deficiency: from diagnosis to the development of a possible therapeutical approach. J Chromatography B, Analytical technologies Biomedical Life Sciences 832: 1-8, 2006.

17. Arata J, Hatakenaka K, Oono T. Effect of topical application of glycine and proline on recalcitrant leg ulcers of prolidase deficiency. Arch Dermatol 122: 626-627, 1986. 